\title{
Interpreting the Paradoxical in the Hispanic Paradox
}

\section{Demographic and Epidemiologic Approaches}

\author{
ALBERTO PALLONI $^{a}$ AND JEFFREY D. MORENOFF ${ }^{b}$ \\ ${ }^{a}$ Center for Demography and Ecology, University of Wisconsin, Madison, Wisconsin, USA \\ ${ }^{b}$ Population Studies Center, University of Michigan, Ann Arbor, Michigan, USA
}

\begin{abstract}
This paper discusses problems that are common to both the epidemiologic risk-factor approach and the demographic variable-based approach to studying population health. We argue that there is a shared reluctance to move away from a narrow variable-based thinking that pervades both disciplines, and a tendency to reify the multivariate linear procedures employed in both disciplines. In particular, we concentrate on the difficulties generated by classical variable-based approaches that are especially striking when one neglects selection processes and the use of strategies to minimize its effects. We illustrate these difficulties in terms of the so-called "Hispanic Paradox", which refers to comparative health advantages that some Hispanic groups appear to have. We find that much of what is conceived by demographers and epidemiologists as a paradox may not be paradoxical at all.
\end{abstract}

KEYWORDS: Hispanic paradox; demographic approach; epidemiologic approach

\section{INTRODUCTION}

In recent years both social epidemiologists and social demographers have grown restless with the dominant paradigms in their respective disciplines. Epidemiologists, for example, have reexamined the risk factor approach to studying population health and its assumptions about the nature of causality, ${ }^{1-5}$ and found it in need of a complete overhaul. Likewise, social demographers have become increasingly interested in the social context of human behavior 6,7 and, with encouragement from their sociologic neighbors, have begun to recognize the severe shortcomings of a purely variable-based view of the world. ${ }^{8-11}$ The roots of this disenchantment with the conventional paradigms in each discipline are remarkably similar and the standard operating analytical procedures in each discipline are also more common than either side is normally prepared to admit. Glaring differences and contrasts do exist but these hold less sway on the outcomes of the research enterprise than do the commonalities.

In this paper we discuss problems common to both the epidemiologic risk-factor approach and the demographic variable-based approach to studying population health. We argue that there is a shared reluctance to move away from an all too easily applicable but narrow variable-based thinking that pervades both disciplines, and a

Address for correspondence: Alberto Palloni, Ph.D., Center for Demography and Ecology, University of Wisconsin, Madison, WI 53706, USA. Voice: 608-262-3131; fax: 608-262-8400. palloni@ssc.wisc.edu 
tendency to reify the multivariate linear procedures employed in both disciplines to estimate effects of conditions or characteristics on outcomes of interest. In particular, we concentrate on difficulties generated by classical variable-based approaches that emerge with full force when one neglects selection processes and ignores the use of strategies to minimize its effects. We then illustrate a manifestation of these difficulties in a problem that has received much attention in both the epidemiologic and demographic literatures: the so-called "Hispanic Paradox", which refers to comparative health advantages some Hispanic groups appear to have on a number of different outcomes including low birthweight, infant mortality, adult mortality, and adult health status. We find that demographers and epidemiologists alike pose this as an ill-defined problem, and that much of what is conceived as a paradox may not be paradoxical at all. We assign much of the blame for the misinterpretation that follows to the limitations of variables-based thinking, shared by both demographers and epidemiologists. We conclude that proper treatment of the Hispanic Paradox, or other similar problems, requires improved research designs and greater attention to the social context of individual actions.

\section{EPIDEMIOLOGIC AND DEMOGRAPHIC APPROACHES TO POPULATION HEALTH}

In this section we define what we mean by variable-based thinking and argue that it leads to important similarities between the demographic and epidemiologic approaches. Although we regard this commonality as a key feature of both approaches, one that may be responsible for our failure to understand certain phenomena, we also recognize that there are important differences that drive a wedge between the two, contrasts in practices that constitute the identity of each discipline, and on which each erects a foundation partly justifying separate disciplinary enterprises. In what follows we first examine differences and then similarities between the two approaches.

\section{Important Differences}

\section{Length of the Causal Chain}

A salient difference is in the content of the variables that demographers and epidemiologists privilege in their approaches to analyzing health status. Epidemiologists tend to focus more on downstream as opposed to upstream variables in trying to predict health conditions. $1,3,5$ That is to say, epidemiologists privilege variables that have a more proximate connection to the health conditions under study and they pay less attention to overall causal process. For example, an epidemiologist may feel content and satisfied after finding that cigarette smoking is associated with a high prevalence of lung cancer. Once statements regarding a measure of relative risk among smokers can be made, the investigator may move on. In fact, it would even be preferable if it were possible to associate measures of quantity or timing of smoking with measures of lung pathology, and then estimate the relative risk of lung cancer among those with various degrees of lung pathologies. Epidemiologists tend to be less concerned with explaining why a non-negligible fraction of all lung cancers 
do not occur among smokers, and why a substantial fraction of smokers may never develop lung cancer. Indeed, to the extent than one attempts to shorten the distance between cause and effect, it becomes less interesting and pertinent to understand the existence of contingencies-why a particular cause exerts a given effect under certain conditions and a completely different effect or none at all under others - or mediation processes - how and through what mechanisms a proximate cause, such as smoking, produces its hypothetical effects.

More distant and removed from the epidemiologist roadmap is the investigation of conditions that lead to smoking or to the adoption of behaviors that may be associated with a pathological outcome. Smokers are not a random sample of the population; smoking is not a trait blindly assigned by an investigator to individuals independently of their characteristics; individuals do not sort themselves randomly into smoker/nonsmoker classes. Smoking initiation is a behavior chosen by the individual and, by the same token, persistence of smoking and adherence to a particular smoking pattern is also a behavior that, to some extent at least, is chosen by the individual. The conditions that lead to more or less heavy smoking and those that are responsible for the more or less uninterrupted persistence of the behavior may not be altogether unrelated to the outcome of interest. These behaviors are in the center of the radar screen of the social demographer who, in turn, pays much less attention to and is much less concerned with the more immediate determinants of the outcome of interest. Thus, when detecting, assessing, and analyzing social class differentials in prevalence of lung cancer, a social demographer will attempt to understand why smoking is more prevalent among those in lower social classes, why quitting is more common among those in higher classes, and why the risk of lung cancer is much lower among high social class smokers than it is among lower social class smokers.

In recent discussions of the nature of epidemiology as a scientific enterprise, some authors have identified this contrast between epidemiology and social sciences $1,3,5$ and advocate the need to embrace an approach to such problems that is more like the one that a sociologist or economist would use; one whereby the researcher constantly tinkers and adjusts the observational and conceptual lenses to allow other phenomena to come into full view. Although there is much to be recommended in this position, we argue below that the manner in which the lens is graduated is not immaterial, and that paying more attention to other phenomena does not by itself constitute a redeeming feature. Quite the contrary, the conceptualization of how other phenomena influence an outcome may also be a conduit for leading the entire research enterprise astray.

\section{Nature of Study Designs and Propensity to Over Control}

A second important and related difference is that epidemiologists tend to privilege a sequential testing or verification procedure that disassembles the object of study into clearly identifiable parts but without following an ex ante plan. This approach proceeds by attempting to isolate the role and effects of one, or at most a handful of factors, without having an a priori formulated model of the relations between them. To the extent that some factor appears to be associated with important effects on an outcome once pertinent factors are controlled for, the enterprise is considered to be on the right course. 
Obsession with controlling for confounding factors is pervasive in epidemiology and determines the utilization of study designs that introduce formidable shortcomings of their own. Thus, epidemiologists prefer clinical trials, case control studies, or rather small community studies, where a number of factors can be exogenously and simultaneously manipulated to large (cross-sectional or panel) population based studies, where many factors change beyond the investigator's control. ${ }^{2,4}$ In general, the study designs favored by epidemiologists are more conducive to the identification rather than the explanation of causal factors,${ }^{5}$ and the price paid is considerable since such designs induce what one may call a propensity to over control for social and behavioral factors. ${ }^{11}$ Efforts promoting close adherence to a paradigmatic ceteris paribus may indeed minimize the role played by confounding factors associated both with outcomes and target factors. However, to the extent that the most proximate determinants of an outcome are intimately related to the controlled factors, inferences drawn in such settings may produce misleading or, at best, sterile propositions. Thus, for example, the effects of smoking obtained after controlling for social class as a "confounding factor" are considered a desirable target. Yet, this net effect can only be interpreted properly at the price of making a higher order abstraction-the idea that we can study the relationship between smoking and lung cancer in a world devoid of the influence of social class.

Being less concerned with mapping the role of single risk factors, social demographers lean heavily toward study designs requiring well-defined target populations, representative samples, a richer stock of characteristics of individuals or social contexts and, if at all possible, panel or longitudinal observations. Because the starting point is usually a set of complex models involving many variables, ex-ante manipulation is replaced by ex-post control, via well-defined multivariate analyses. This is not to say that such study designs are optimal. Far from it, the indiscriminate and uncritical utilization of multivariate models offers as many opportunities for misleading or even meaningless inferences as do pure case control studies.

\section{The Role of Time and History}

A third contrast separating social demographers from epidemiologists is the concern for time and history that permeates the work of the former and that seldom enters into the work of the latter. Time, after all, is simply an opportunity for other factors to intrude into the picture and disturb the operation of selected single risk factors. Thus, it is not an exaggeration to say that, from the point of view of conventional epidemiology, study designs in which the passage of time is controlled for are more desirable than those where the passage of time is variable across individuals. Time is only of interest as a proxy measure for degree of exposure to a risk, because in most cases what matters for an outcome is the accumulated risk rather than the risk at any one instant in time. Thus, for example, age features prominently in epidemiologists' models as a proxy for the passage of time. Time cum exposure is one of the few permissible roles for time in epidemiology or, at least, the only role where it is taken seriously.

The openness to causal processes that are complex affairs is perhaps responsible for a different attitude toward time that prevails among social demographers. Time enters into their purview in a number of ways. It may take the form of (1) short-term lagged effects of independent (or dependent) variables in autoregressive or ARIMA 
models, (2) longer-range historical or developmental characteristics assessed far before the period when the outcome or other covariates are measured, (3) controls for explicit events that took place during past historical periods and thus introduced to capture period effects, or (4) measures that assess the degree of membership to a particular cohort. After all, it is in demography, not epidemiology, where the explanatory power of cohort began its ascent and where it acquired dominance, ${ }^{12}$ regardless of whether cohorts are powerful determinants of behavior. ${ }^{13}$ More recently, time has emerged as an all intrusive force, as models for survival analysis (event histories) require that the analysts consider complex chains of events and do away altogether with sedating Markovian assumptions (e.g., the limitation of historical influences to only the immediate past and the homogeneity of effects across time and space).

Although far from constituting the tools for a perfect placement of individuals or other social units into their proper historical contexts, the procedures used by social demographers have, by and large, been more sensitive and better programmed than those employed by epidemiologists to incorporate the role of history, and to regard the passage of time as more than innocuous, a nuisance to dispose of unless it is a marker of cumulative exposure.

\section{Different Deployment of Similar Methods}

Finally, although in appearance similar, the methodologic machinery utilized by epidemiologists and social demographers is quite different, if not in its roots, at least in its concrete applications. A quick glance at research papers published in the main journals associated with each discipline or, alternatively, an examination of the curricula of their respective graduate programs, reveals a stark contrast in methods employed. In epidemiology most analysts focus on measures of risk embodied in odds ratios and relative risks, whereas social demographers center their attention on unstandardized measures of effects, the high-profile regression coefficients. On the surface these are similar metrics, statistics whose calculations require similar operations, whose values are determined by similar empirical relations, and whose interpretations rest on like assumptions and obey comparable distributional laws. However, these apparent similarities conceal important differences. First, interpretability of regression coefficients in social demography rests heavily on complex models that, more often than not, consider simultaneous causality and incorporate network of causal relations (simultaneous equation models), recognize latent constructs made up of multiple indicators (latent and structural equation models and models with multiple indicators), and involve nested processes (choice based models, selection models). Instead, odds ratios in epidemiology emerge from and are couched in the most simplistic of linear representations, with little concern for the existence of simultaneity, nested processes, sequence of causal stages, and potential differences between latent constructs and indicators.

Second, whereas in epidemiology odds ratios and relative risks are viewed as tools to rank order risk factors, social demographers are more likely to use regression coefficients to help allocate variability of an outcome attributable to factors in the model. It is through these allocation operations that the social demographer can break down total effects into component parts, namely, background characteristics, mediating factors, and contingencies (interactions). 
There is more. Just as modern epidemiology is obsessed in its quest for identification of prominent risk factors for just about all conditions it studies, social demographers pursue relentlessly the decomposition of effects not just across factors ordered in a causal hierarchy but also across what we may call "propensities" (measured as rates) and "population composition." This is the primary role of elementary standardization techniques, but it is also the sole objective of even the most sophisticated linear models as well. The closest one comes to this approach in epidemiology is through calculation of population attributable risks (PAR); but PAR is a poor cousin of full standardization as its sole purpose is to quantify a (sometimes unattainable) counterfactual, rather than to enact a fully specified causal scenario. Both PAR, as used in epidemiology, and classic standardization and decomposition techniques, as used in social demography, are to be faulted on the grounds that they are frequently uncritically applied, and that their outcomes are too often reified without regard to the weakness of the assumptions that permit their operationalization.

\section{Similarities Between the Two Approaches}

Despite the aforementioned differences and contrasts, the two disciplines are unified when embracing a variable- or risk-based approach that imposes a number of important constraints on interpretations. These, in turn, result in a several shortcomings. We tread familiar territory here, since some social scientists $8,10,11$ as well as epidemiologists ${ }^{1,5}$ have already paved the way for what turns out to be a devastating critique of the variable-based approach. Our aim is to identify some of the most strategic problems and then illustrate the complications they cause using the Hispanic example.

The variable- or risk-based approach starts with the existence of units (individuals, organizations, groups, etc.) that possess attributes or properties that may, through some set of relations to be specified, influence the occurrence of some other attribute or outcome, also characteristics of the unit of observation. The specification of relations usually takes the form of a linear model-that is, one where the effects of given attributes enter linearly and where the outcome or dependent variable is observed up to an error term. The latter is considered to be independent of all attributes and unrelated to those associated with the outcomes of any other unit. The drill is well-known: the researcher employs the observed variance-covariance matrix to derive estimates of regression coefficients (or odds ratios); these are then interpreted as measures of the outcome's response to counterfactual changes in the attributes of interest. However, this interpretation comes at the cost of making assumptions that have problematic implications for both demographic and epidemiological research. These problems include dismantling the object of study, accepting an inflexible causal interpretation, neglecting the order of events, and distorting social context.

\section{Wholesale Dismantling of the Object of Study}

Attributes (i.e., variables) must be separable from each other and their operation must be independent of the particular dimensions that define any concrete unit or individual, whether these attributes are included in the model or not. ${ }^{8,10}$ Attributes have an existence quite apart from the units they presumably describe so that, for example, a given occupation or educational level is assumed to mean the same thing 
in two individuals, net of other equally separable attributes, and regardless of how they may have been attained by these individuals. This assumption is also troubling in the case of measuring ethnic-group membership. For example, researchers may use a variety of criteria to classify Hispanics or Hispanics of Mexican-origin, such as self identification, residence in a household where the head self identifies as Hispanic, or by means of a Spanish surname. Thus, the content of the attribute changes depending on how the researcher measures it.

Quite apart from slippery definitional content, being Hispanic or Hispanic of Mexican origin, is still an abstraction. First, it means quite different things in different geographic locations in the U.S., since the process whereby individuals become part of the category (illegal or legal migration, being born to or adopted by a migrant family) are completely different. Second, higher order abstractions such as Hispanic of Mexican-origin with a given level of education - the abstraction that helps us interpret effects of education controlling for ethnicity - are conceptually manipulated as if they were unproblematic when, in fact, they are quite so. This is because the assumption invoked to facilitate the interpretation of the estimated effects of education-that highly educated Hispanics are like other Hispanics except for their level of education - could be patently absurd if the paths that allowed them to reach different education levels were made possible by choices and characteristics that could have some bearing on the processes we are studying.

To the extent that individuals are seen as fixed combinations of attributes and characteristics, the interpretation of estimated effects - as variation in an outcome when one and only one attribute is changed-is unassailable. The problem is that the counterfactual on which the interpretation rests is, for all purposes, meaningless until we can convince ourselves that changes in an attribute can take place in an individual without other attributes changing as well. Individuals change as their properties and attributes change, and the quantity and quality of these changes depend on the value of and relations between other attributes. Thus, the estimated effects refer to individuals who do not exist and are unlikely to ever exist. After disassembling the individual to permit assessment of net effects, we are not in a position to reassemble them since they are not the simple addition of attributes and characteristics included in the linear model. This limitation of the variable approach is examined in more detail below with an example that recurs in the study of the so-called Hispanic Paradox.

\section{Inflexible Causal Interpretation}

A second problem of the variable approach is that it induces and promotes a heavy-handed interpretation of causal effects. Estimated effects are assumed to be unique, rather than part of a vast multiplicity of possible ones, as they are often times portrayed in theoretical representations. Thus, the direction and magnitude of causal effects associated with some attributes are assumed to be identical across individuals. This may not be so. Effects can change their direction and magnitude depending on the social contexts where individuals reside, on the histories of individuals, and the interaction between these two. The direction of causality itself may be reversed depending on social context and historical trajectory.

Admittedly, one may argue that difficulties derived from inattention to this problem are more a result of laziness or lack of industriousness among researchers than 
a shortcoming inherent to the variable-based approach. In fact, the general linear model does offer plenty of opportunities and tools for retrieving high-order interaction effects and, when feasible, the study of interaction effects is the richest part of the analysis of social phenomena. However, the operations needed to study highorder interaction effects have shortcomings of their own, mostly associated with limitations already described, namely, that characterization of social contexts and of individuals histories must proceed via the variable approach and, therefore, must fall prey to the traps involved in the characterization of social context or historical trajectories via abstract properties or attributes. ${ }^{10}$ There are other limitations inherent in the variable-based approach that make it difficult for the researcher to identify high-order interactions. These are numerical difficulties entrenched in the procedures employed to calculate estimates of effects: posing several layers of interaction effects quickly wears out the observed realization of an outcome, strains the numerical evaluation of variances and covariances and, ultimately, imposes severe limits to the depth of the causal bundle that one may be in a position to study.

Neglecting Order of Events

A third problem is that a reified linear approach completely overlooks that the sequence of past events may, on its own, have a powerful influence on the occurrence of individual attributes. ${ }^{9,14}$ In other words, it is not just the distribution of values of certain attributes today or at some point in the past that matters but the exact (or approximate) sequence of events through which such distributions came into existence. The introduction of survival analysis has made it possible to retrieve some of these effects but, as it turns out, it is too blunt an instrument to get at the heart of the matter. Sequence analysis has been proposed by some researchers as an alternative, ${ }^{14,15}$ but is too early to say whether this tool holds any promise or is just a passing fad. For the time being, in the absence of models reproducing the effects of event sequences, we have no other solace than to be aware of the problem and not to overinterpret the results from models that do not have any place for historical sequences at all.

\section{Distortion of Social Context}

A fourth problem with the variables-based framework is that it introduces assumptions that distort the social context of individuals. These assumptions relate to problems of selection, endogeneity, and the effect of social context on individual behavior.

Selection and Endogeneity. Individuals' histories of past decision-making and past behavior constrain the range of characteristics or attributes they may possess at any given time. To the extent that we ignore such processes when they are related to the outcome we study, we will be violating one of the main assumptions of the linear model. Many violations of this sort fall under the rubric of selection processes or, more generally, of endogeneity biases. Although there are ad hoc statistical solutions to these problems, these are no more than guides that signal a lack of robustness or the extent to which our estimates may have gone astray. Real solutions require more direct consideration of the neglected processes.

Social and Contextual Effects. The imagery of independent units of observations in a sample (another foundational assumption of linear models) is often just that, 
an image. In many cases it is a misleading one. This is because the very characteristics that individuals possess - for example, ethnicity, membership in a communitysubjects them to common influences, including the influence of other individuals' actions. The idea that individual behavior is a function of social context is a very old one, but its full integration into conventional modeling has taken a long time to occur. Even now, the way in which we solve the conundrum is not satisfactory. Hierarchical linear models (HLM) solve some of these problems but, in truth, they are illequipped to deal with some thorny issues such as those involving mutual influence of individuals. ${ }^{16,17}$ This problem has been most seriously studied in the literature on social networks, but to our knowledge this literature has had only meager spill-over effects on the rest of the social sciences or epidemiology. ${ }^{18}$

More Endogeneity. Once we introduce social effects we open the door to an additional difficult problem. The variable approach is not concerned with the possibility that individuals may choose their social contexts or that some of the characteristics of the communities where individuals reside are in fact a function of individuals' characteristics themselves. Thus, for example, decision making about location of services in neighborhoods is, at least in part, a function of observable properties of the individuals living in such neighborhoods. Here causality flows, as it were, in a direction opposite to the one normally posed, and moves from the individual to the aggregate rather than the reverse.

\section{Disciplinary Commonalities and Contrasts}

The problems highlighted above point toward an unavoidable result, namely, that the variable-based approach dismantles the individual in order to secure meaningful inferences. The price paid for identification and evaluation of risk factors is that we lose sight of the entire causal process and instead focus on relations between abstract attributes. Can the whole be reassembled? Can the variable or risk approach put back together what was dismantled for analytical purposes? The tools for doing so are primitive and our experience with them too scarce to be of much value. Suffice it to say here that standardization and decomposition techniques are typically the preferred tools chosen by social demographers to deal with this problem, but these are deployed far less frequently than would be necessary to restore some of the richness to the causal processes being studied.

We do not mean to detract from the formidable power associated with a properly used variable-based approach. After all, many important discoveries in epidemiology, demography, and social sciences in general owe their existence to the deployment of such an approach. We only intend to emphasize that the relaxed or uncritical use of such an approach may sometimes magnify more than necessary the influence of its shortcomings, and that the only way to avoid such a state of affairs is to be aware of them.

With this review of commonalities and contrasts we hope to have shown that epidemiology and social demography have a great deal in common, more than what it is normally thought to be the case. In what follows we attempt to show that the relevance of such commonalities is more consequential than the relevance of the contrasts. To do this we examine the nature of a very concrete research problem, the socalled Hispanic Paradox, which has been studied by demographers and epidemiologists alike. Indeed, we argue that some of the problems associated with the resolution 
of the so-called Hispanic Paradox may have been circumvented altogether had researchers paid more attention to the aforementioned difficulties inherent in the variable-based approach.

The following three sections illustrate the role played by weakness in the variable-based or risk-based approach in the case of the Hispanic Paradox as it pertains to infant mortality and to low birthweight. We trace the history of the Paradox, summarize the evidence marshaled to build a case for it, and discuss the most glaring problems that analysts must face to formulate the problem properly. In a subsequent section we show that similar considerations apply to the Hispanic Paradox as it pertains to adult health and mortality.

\section{THE HISPANIC PARADOX: OVERVIEW AND ASSESSMENT}

\section{Definitions}

The Hispanic Paradox is a remarkably slippery idea, a moving target of sorts that refers to a number of very different things. The concept is composed of three dimensions, all of which are subject to change and do indeed change over time and across researchers. The first dimension refers to the outcome of interest. This can be one of the following: (1) infant and child mortality, (2) adult mortality, (3) birthweight, or (4) adult health status. The second dimension refers to the target population and this can be one of the following: (1) the Mexican-origin population (born in Mexico) but residing in the U.S., (2) Hispanic-origin population (born in Mexico and other countries in South and Central America) and residing in the U.S., or (3) the Spanish surname population residing in the U.S. Note that the last group includes a mixture of individuals born abroad who migrated to the U.S. and some but not all of their descendants. The third dimension refers to the contrast population or the population used as a standard to compare outcomes observed in the target population and to adjudicate whether differences on some outcome are worthy of the label "paradox". Sometimes the standard is (1) the non-Hispanic white population and others (2) the non-Hispanic black population. In all cases, Puerto Ricans born in the U.S. or abroad are not considered part of either the target or the standard populations. ${ }^{19}$ In fact, for the most part, this group represents an exception to the Hispanic Paradox when this is inferred from contrasts between any of the standard populations mentioned above and a target population including the Hispanic-origin population. Thus, in theory at least, there are 24 possible contrasts or comparisons that may be used to produce inferences about the relative health status or performance of the groups involved. Although the literature on the subject has covered them all, only a few stand out as rich and promising. These are contrasts relating to infant and child mortality, adult mortality, and birthweight (three outcomes), where contrasts focus on the Hispanic population, however defined (three possibilities), and employing either the non-Hispanic white and black populations as standards. This reduces the comparison space to at most 18 . Since the non-Hispanic white population is more frequently used as standard ${ }^{20-38}$ we have only nine possible comparisons for inferences. To reduce even further the space of possible comparisons, we choose to focus on infant mortality and birthweight only. Our review of the literature pertaining to adult health and mortality leads to similar conclusions but, because the history of the paradox associated 
with these outcomes is of more recent origin and research on it more sparse, it does not lend itself to as rich a treatment as do infant mortality and birthweight.

\section{Brief History of the Hispanic Paradox of Infant Mortality}

The most interesting and thorough review of the Paradox, at least the one emerging when comparisons are confined to infant and child mortality, was written by Gutmann and colleagues. ${ }^{21}$ In this review the authors trace the origin of the Paradox to the work of Teller and Clyburn, ${ }^{20}$ who estimated rates on infant mortality for the Spanish surname and non-Hispanic white populations, based on special vital statistics tabulations for Texas in 1964. They found that infant mortality rates for the former were "only slightly lower than that of non-Hispanic whites". ${ }^{20}$ Vital statistics data for 1972 revealed that the two groups were not only closer than estimates for the past showed them to be, but they also indicated that the neonatal mortality rate was more favorable among the Spanish surname population. This, in sum, is the set of findings that constitute the original Hispanic Paradox.

Two remarks about this original research are necessary. First, the target population, defined by Spanish surname, includes both U.S.-born Mexican Americans and Mexican immigrants. It also could also include many individuals from countries other than Mexico, but there were unlikely to be many of them living in Texas circa 1964. Second, the outcome variable in the original Hispanic Paradox was constrained to be infant mortality, with occasional distinctions between neonatal and postneonatal mortality.

More recent studies have examined the history of the paradox with data from Bexar County, Texas dating back to $1935,{ }^{29}$ a 1900 Census microsample on six Texas counties, ${ }^{21}$ the 1910 U.S. Census national microsample, ${ }^{21}$ and the 1990 linked birth and infant death files. ${ }^{21}$ This work has generally shown that the Paradox is a phenomenon of relatively recent vintage. For example, the infant mortality rate of the target population (variously defined as Hispanic, Mexican-origin, or Spanish surname) was substantially higher than that of whites in both the 1900 six-county sample in Texas and the 1910 national sample. ${ }^{21}$ Moreover, in the 1900 national sample, the target (Hispanic surname) population rates were also 33 percent higher than those of blacks. The historical data from Bexar County, indicate that the infant mortality rates of the target population (Spanish surname) began to converge with those of non-Hispanic whites in about $1960 .^{29, c}$ However, Bexar County data from 1975 to 1984 reveal that infant mortality rates were still 22 percent higher for the target group (Spanish surname population) than they were for non-Hispanic whites. ${ }^{21}$ The 1990 national data show that infant death rates are almost identical for the target group (Mexican-origin) and non-Hispanic whites and are higher among blacks. ${ }^{21, d}$

In sum, the available historical data seem to indicate that the Hispanic Paradox, if it exists at all, did not emerge until approximately 1960. Since then, it has consisted of a smaller than expected difference in infant mortality between the target group

\footnotetext{
${ }^{c}$ The convergence between the non-Hispanic white and Spanish surname populations in Bexar County occurred around 1940 for rates of neonatal mortality. ${ }^{29}$

${ }^{d}$ An important feature of the findings from the 1990 data is that when SIDS deaths are excluded from the infant mortality rate-or when infant mortality is defined as exogenous mortality - rates for the Hispanic population are $26 \%$ higher than they are for non-Hispanic whites. Thus, the paradox also appears confined to endogenous (mainly neonatal) infant mortality.
} 
and the primary standard group (non-Hispanic whites) and of a widening advantage favoring the target group when they are compared with the secondary standard group (non-Hispanic blacks).

The notion of an "expected" difference between the target and standard populations reveals the limits of variable-based thinking. It is an abstract construct associated with an implicit belief that Hispanics should have worse health outcomes than non-Hispanic whites and perhaps non-Hispanic blacks. Thus, even when some recent estimates for Bexar County show that Hispanics are at a disadvantage with respect to the non-Hispanic white population, such differences are cast as being "smaller than one would expect". The virtual parity between the two populations in the 1990 data set appears to more clearly confound such expectations, as might the fact that Hispanic do better than blacks on infant mortality in both the national and Bexar County data sets.

These empirical facts are paradoxical only if one holds on to the idea that the target population has a considerably more adverse risk profile than the primary or even the secondary standard populations. In other words, the Paradox depends on the idea that the target population is a high-risk group, a view that is supported by some evidence that Mexican Americans have "lower levels of education than African Americans and are less likely to receive adequate prenatal care or have medical insurance to defray costs of delivery than are African Americans" (Bean and Tienda ${ }^{39}$ cited in Gutmann et al. $\left.{ }^{21}\right)$. Other conditions affecting the risk of neonatal and postneonatal mortality, such as maternal age, parity, and birth intervals, are also believed to be adversely distributed among the Hispanic population. However, many of these propositions are not firmly grounded in empirical findings. For example, an examination of a long series of CPS data does not show that the target population (defined either as the Hispanic or Mexican-origin population) is uniformly worse off than nonHispanic blacks or even non-Hispanic whites on a number of risk factors. ${ }^{40}$ Similarly, information from NCHS, NSFG, and NIMHS reveal that on some risk factors, Mexican-origin individuals appear to have a disadvantage, but on others they clearly have an advantage over other groups. ${ }^{41}$ On the whole, we do not find here the necessary evidence to support the argument that uniform differences put Hispanics of Mexican-origin at a distinct disadvantage.

Nevertheless, if one accepts the idea of the paradox, then it would suggest the following corollary: when factors defining the pertinent risk profile of each population being compared are controlled in a multivariate analysis, one should find that the relative standing of the target group improves vis-à-vis that of non-Hispanic whites and blacks. This would be true because presumably the factors being controlled represent disadvantages for the target group. This corollary, of course, holds true only if we also assume that the effects of the factors contained in the risk profile are identical across groups.

The evidence from the studies reviewed above is mixed on this proposition. On the one hand, data from the 1990 linked birth and infant death records partially analyzed by Gutmann and colleagues ${ }^{21}$ (see their Table 2) support this corollary since the odds ratios for Hispanic infant deaths drop from 1.03 down to 0.94 after controlling for maternal education (and other factors less germane to maternal and infant the risk profiles). However, this change is only minor suggesting that differences in risk factors across groups are not very important. (Also, when the standard is the black 
population there are no changes in the relative odds-it remains pegged at 0.48.) The results from the Bexar county data are somewhat more convincing since in all four decades under examination the introduction of control variables reduces the white advantage over Hispanics in infant mortality, although whites still maintain an advantage. On the other hand, results from the 1910 microcensus sample (see Table 5 in Ref. 21) go in the opposite direction: the Hispanic-non-Hispanic white ratio for child mortality increases after controls and so does the Hispanic-black ratio. Regardless of what the target population is, there is no relative advantage before or after controls. In sum, these bits of evidence concerning the direction of estimated differences when adding controls do not definitively support the corollary formulated above, and so the very idea that a Paradox exists is shaky from the outset, although it is supported more strongly by the more recent data.

However, what is permissible to conclude from this evidence? It is difficult to disagree with the first conclusion drawn by Guttmann and colleagues, namely, that the Paradox regarding the contrast between targets and non-Hispanic whites came into existence only during very recent times, perhaps as late as 1960-1970. Given the evidence summarized previously, it is considerably more difficult to agree with the other conclusion, namely, that early on there was only one Paradox and it was that "poor Mexicans were as well off or better than their black neighbors." First, the Hispanic health advantage over blacks is not exactly ubiquitous in the historical record and, second, the magnitude of the Hispanic-black gap remains the same or diminishes significantly when controls are introduced suggesting that, if anything, blacks have a more disadvantaged profile than Hispanics, rather than the other way around. So, there is no paradox there. What does resist this kind of scrutiny are the recent contrasts between the target group and blacks (favoring the former) and between target group and non-Hispanic whites (favoring the latter, but by a less than the "expected" margin). In fact, this inference from recent trends is not just more palatable but also somewhat immune to the charge that we could level on the inferences drawn from historical trends, namely, that the assumption of invariance in the principles used to classify the target group is questionable.

\section{Explanations}

For the sake of argument, let us accept the idea that the observed contrasts in infant mortality rates are paradoxical, especially the patterns emerging in the more recent data. What kind of explanations have been offered, and can these explanations account for the fact that the phenomenon is of rather recent origin? The most frequently offered explanations for the Paradox in the literature are (1) the underregistration of infant deaths in the target population, (2) differences in the maternal risk profile, (3) differences in maternal behaviors, (4) effects of social networks, and (5) the healthy migrant hypothesis that immigrants represent a selectively healthy group that are not representative of the wider target population.

The first explanation has been dismissed outright, but probably too quickly and hastily. We are not aware of any large-scale effort to actually test this hypotheses, at least not in a way that is comparable to what has been done for the case of whiteblack differentials in adult mortality in the U.S. ${ }^{42}$ Instead, we find assessments of some data sets (e.g., Bexar county) where the authors assert that errors of underregistration could not reasonably be large enough to account for the observed patterns 
of infant mortality differences. ${ }^{29}$ Admittedly the claim for that particular data set may be compelling but as a general explanation does not have the power to close the case once and for all. The important point is that this alternative explanation has not yet been excluded and may, together with other problems, undermine the case for a paradox.

The second explanation-differences in maternal risk profiles-is not really an explanation, because if Hispanic women do have a risk profile conducive to higher infant mortality, then these risks would reinforce the Paradox, not explain it. Indeed, as argued above, standardizing for conditions thought to be related to infant mortality may either exaggerate, attenuate, or leave invariant the contrasts between groups, and where changes are observed they are usually inconsequential.

The third candidate-differences in maternal behaviors-is the most widely accepted explanation. As Gutmann and colleagues 21 argue: "Perhaps the most promising explanation to emerge is the finding that women of Mexican-origin are characterized by a more healthful lifestyle that buffers or offsets disadvantaged sociodemographic conditions. For example, Mexican-origin women smoke less, consume less alcohol, and have more nutritious diets than do non-Hispanic white women." This argument holds that the target group's advantage over non-Hispanic whites and blacks is rooted in maternal attributes that are (1) strongly related to conditions affecting neonatal not postneonatal mortality and (2) acquired in social and normative contexts that are very different from those they encounter in the U.S.

The fourth explanation - differences in social networks - has a very similar logic: mothers of Mexican-origins retain active linkages to and membership in strong social networks that help cushion and offset the potentially deleterious influence of stressors. ${ }^{43}$ As was the case with behaviors, networks are assumed to be rooted in the context of the country of origin and are expected to weaken with length of stay in the U.S.

There is much to recommend these two explanations, since they are consistent with three observed regularities. First, these factors are invoked predominantly as determinants of mortality during the first month of life and are not thought to influence to any significant extent the postneonatal period. This fits with the fact that the Paradox does appear to be rooted in comparisons of neonatal mortality rates. Second, the factors alluded to in these explanations are linked to neonatal mortality through their affect on birthweight, and it is the case that there is a related Hispanic Paradox pertaining to birthweight as an outcome (discussed below). Third, because these explanations apply more strongly to neonatal mortality, they appear more plausible in more recent time periods, when postneonatal mortality ceased to be an important component of infant mortality. Thus, these explanations may also address the question of why the Paradox did not appear until relatively recently. ${ }^{21,29}$ It is also possible that, over time, group disparities in pertinent behavioral profiles between the target group and non-Hispanic whites (or black) have widened, further strengthening this position.

Although these arguments constitute a solid scaffold supporting the case for the Paradox, some problems still persist. First, to echo an earlier theme, even in the very careful work of Gutmann and colleagues ${ }^{21}$ and in that by Forbes and Frisbie, ${ }^{29}$ the target groups are not, and cannot be expected to be, the same-the definitional changes from Spanish surname (particularly in the earliest formations), to Hispanic 
origin or Mexican origin, and combinations thereof. This weakness is a problem inherent in the variable-based approach: abstract attributes are often not generalizable over time and space.

In this case, the comparisons involve very different groups and open the door for strong counter arguments. For example, the definition of the target group never fixates on foreign-born or U.S.-born women. Yet one of the facets of the Paradox appears to be that compared to immigrants, second-generation Hispanics have rates of infant mortality that are less similar to non-Hispanic whites and more similar to non-Hispanic blacks. This inconsistency causes a problem. One popular explanation for the decrease in the outcomes of second-generation Hispanics is acculturation and the adoption of behavioral profiles that depart from those adhered to by the Mexican(or Hispanic-) origin population. ${ }^{44}$ If the acculturation hypothesis were true, however, it should be the case that they should attenuate or disappear (1) with the passage of time (as the second generation grows), (2) when the target group is restricted to second-generation Mexicans, and (3) when the target group is restricted to those who have lived longer in the U.S. (or are more spatially assimilated within the U.S.). As far as we know, none of these inferences has ever been put to a test despite the fact that they are a recognizable part and parcel of the central argument.

The final explanation is the healthy-migrant hypothesis. Much like the under registration of deaths argument this explanation has been rejected somewhat hastily without a closer look of the available evidence. We examine this hypothesis in great detail below, but for now we suggest a thought experiment that should challenge the typical knee-jerk reaction against it. If one rejects the healthy migrant explanation in favor of a behavioral or network-based explanation, it follows that the neonatal mortality rates observed among Mexican immigrants in the U.S. should be comparable to what would have been observed among the Mexican-origin population had this population remained in Mexico (the same argument could be made for other Hispanic ethnic groups as well). Indeed, their behavioral profile as well as their ability to draw resources from social networks (the two factors to which primary causality is attributed) are thought to be part of the cultural and normative context of their country of origin, not an attribute acquired in the U.S. However, the implications of this inference are at odds with the evidence: the levels of neonatal mortality in Mexico circa 1990 are of the order of 26.9 per 1,000 live births, implying a postnatal rate of about 20.6 and an infant mortality of about 47.5 (Demographic and Health Survey reports, several years). These values are at least nine times as high as those among the Mexican-origin population in the U.S. during the same time period. This is a remarkable difference, and the only way to explain it is with reference to factors that account for a large fraction of the difference between the observed and expected levels of neonatal mortality for Mexican-origin population living in the U.S. but that are also wholly irrelevant to any of the standard populations (i.e., non-Hispanic whites and blacks). Prior theory and research does not offer any grounds to speculate on what these factors may be. In fact, one of the most potent behavioral determinants of neonatal mortality, breastfeeding status, is likely to be more favorable in Mexico than in the U.S., so it cannot be a candidate. In the absence of such alternative explanations, it seems plausible that the comparison between Mexican-origin women in the U.S. and those living in Mexico is an inappropriate one, because of various selection processes. We return to this theme below. 


\section{THE PARADOX OF LOW BIRTHWEIGHT: WHAT IS THE EVIDENCE?}

Although the history of the paradox regarding incidence of low birthweight is of more recent origins than the original infant mortality paradox, the two are inherently connected because the bulk of variation in neonatal mortality is accounted by birthweight alone. In this section we examine the empirical support for this facet of the Hispanic Paradox. As was the case with infant mortality (and particularly for neonatal mortality), we find that the evidence, though not overwhelming and often times flawed, shows a difference (albeit a small one), in the incidence of low birthweight between the target population and non-Hispanic white and black groups. The target group compares favorably to non-Hispanic blacks, but the comparison with non-Hispanic whites sometimes favors whites and other times favors the target group, depending on the data set and the way the outcome is specified-that is, a dichotomous measure of low birthweight versus a continuous measure of birthweight. ${ }^{41}$ The evidence supporting this paradox is fragile, but it cannot be dismissed offhand.

\section{A Brief Review of National Level Data}

Data for the period 1980-1997 from U.S. Vital Statistics show that the percentage of low birthweight among Hispanics in general and the Mexican-origin population in particular are within the range 6.1 to 6.5 , whereas rates of low birthweight range from 5.7 to 6.5 among non-Hispanic whites and from 12.7 to 13.5 among nonHispanic blacks. ${ }^{41}$ FIGURE 1 displays group disparity ratios for the period 1980 to 1997, defined as the ratio of the low birthweight rate in a particular group to the low birthweight rate for non-Hispanic whites. A ratio of 1.0 in the graph reveals parity with the non-Hispanic white group. As in the case of infant and neonatal mortality, the Paradox is precisely the unexpected parity between the target group and the standard group. The Mexican-origin populations experience virtual parity with whites throughout the almost twenty years spanned by the data. In fact, they appear to fare marginally better in the last five years of the period, a result of recent increases in the proportion of low birthweight among non-Hispanic whites. However, Puerto Ricans experience less parity than any other Hispanic-origin group. The ratios displayed in FIGURE 2 generalize the Paradox. This graph shows that parity and outright advantages favoring Mexicans relative to non-Hispanic whites hold up after controlling for maternal education. Indeed, not only do Mexican-origin women with low education experience lower incidence of low birthweights than their equal in education in the standard group, but their relative advantage is more marked than those with higher education. In general, however, the difference between Hispanics (excluding Puerto Ricans) and non-Hispanic whites are minor, whereas those relative to non-Hispanic blacks are quite large.

\section{A Review of a Sample of Studies}

To some extent at least, these findings, grounded on national vital registration data, echo and reproduce findings reported in studies with a narrower geographic scope. To summarize the results in the literature, we reviewed close to 50 studies published in the last 10 years in epidemiological, public health, demographic, and 


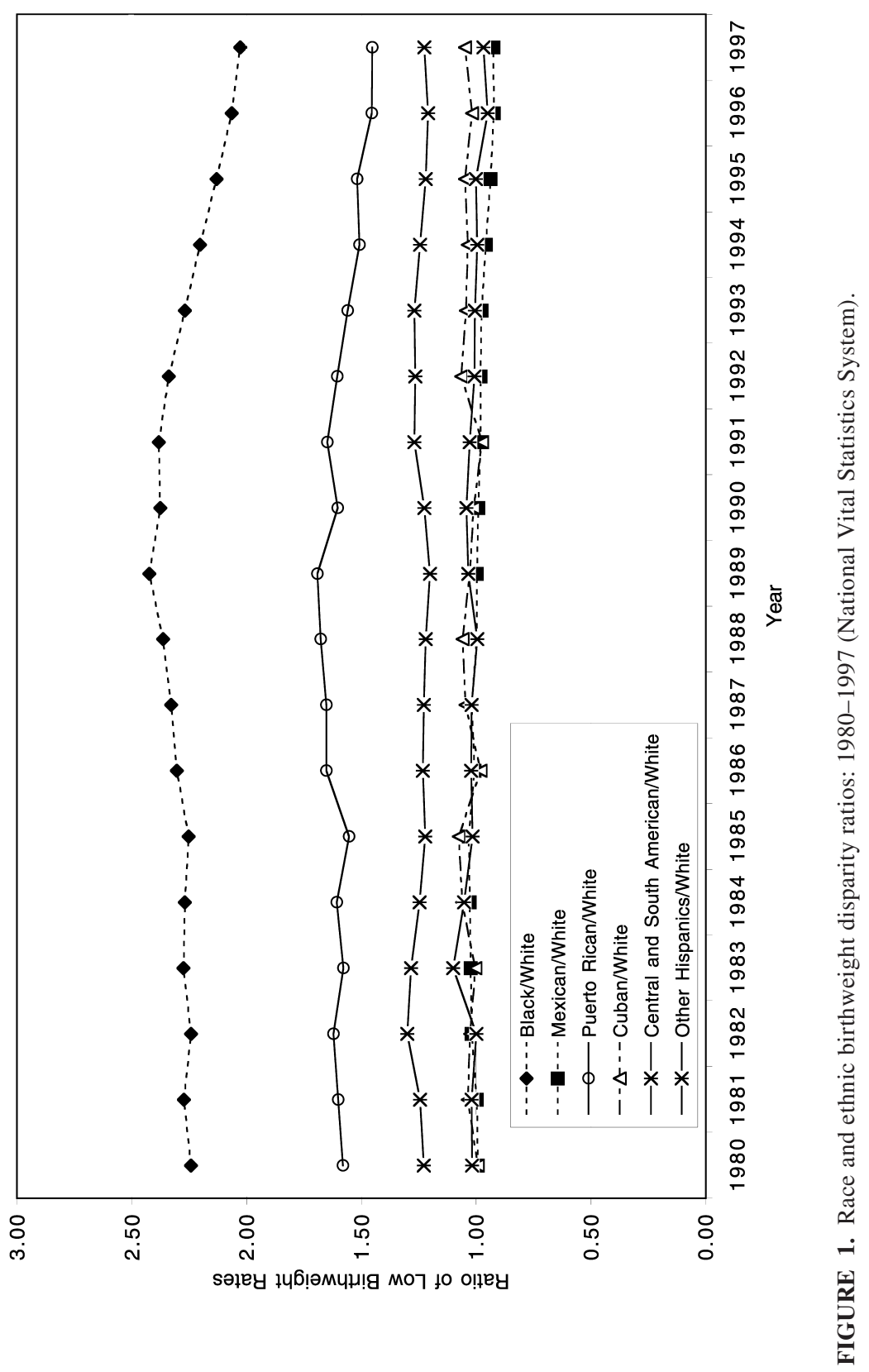




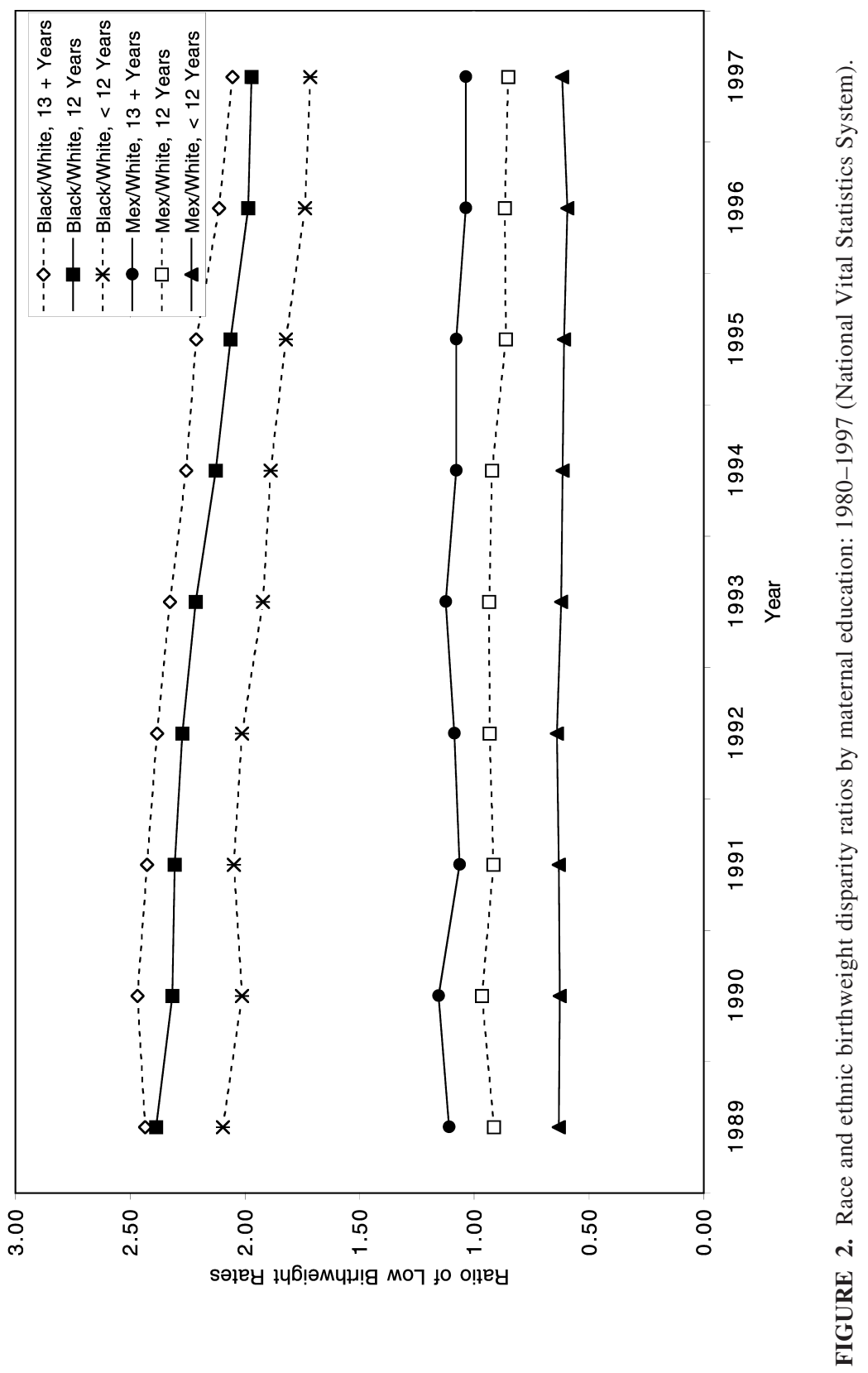


sociologic journals. Our review reveals a bewildering variety of research designs, some based on representative samples of the U.S. population, but most relying on small samples of non representative groups or communities. The studies differ regarding the target and standard populations, definition of proper controls or independent variables, measurement of effects and formulation of basic initial models. In all cases, the research endeavor revolves around comparisons of incidence of low birthweight in a target and a standard population. With a handful of exceptions, estimated regression coefficients or odds ratios are the main statistics used to draw inferences regarding the target and standard populations are made. In only a few of these studies is there an explicit preoccupation with the characterization of the target population, and in none is there an attempt to compare the target populations with sending populations located in countries other than the U.S.

Although this review is informal, its results can be unambiguously interpreted and are likely to be confirmed with a more rigorous meta-analysis. ${ }^{6}$ Our main conclusions can be summarized as follows:

i. The evidence supporting the existence of a Hispanic Paradox is drawn from an exceedingly heterogeneous set of studies, with contrasting outcome measures, definitions, samples, target populations, methods and levels of sophistication. We find that the Hispanic Paradox is not universally verified, that the strength of the underlying relations (effects of ethnic group on the probability of low birthweight) varies across space and time, that its magnitude is modest, and that the estimates can seldom be unequivocally interpreted due to inappropriate controls.

ii. In most cases it is unclear what the nature of the target population is, beyond the particular target group used in the sample. Of particular importance is that results vary, not just depending on the definition of the target population, but also as a function of the geographic location of such groups. This is not surprising. If, as we argue later, there are powerful selection effects accounting for some of the "unexpected" findings, it stands to reason that the outcomes of interest will vary sharply by region of residence. As a consequence, one should expect and not be surprised by the fact that contrasts between target and standard population include important geographic heterogeneity.

iii. In all cases standard linear models are applied and a somewhat rigid adherence to its tenets is maintained. As a result, studies end up overinterpreting the categories used to pigeonhole individuals (ethnic groups) and excessively simplifying the social processes they ought to be interpreting. This includes but is not reduced to: bypassing the conceptualization of complex causal relations and high-order interaction effects, neglecting or mishandling issues related to social selection and endogeneity, ignoring the social history of the populations involved, and overlooking or downplaying the role of social effects which, according to extant migration theories, ought to be ubiquitous.

\section{A Disturbing but Persistent Counterfactual}

In the large majority of cases researchers interpret the findings about birthweight as if they indeed revealed a paradox. The explanations invoked are also virtually identical to those offered to explain the Hispanic paradox in neonatal deaths, placing an emphasis on the presence of either favorable maternal behavioral profiles or 
efficient social network support among Hispanics. However, as was the case with infant mortality, researchers do not pose the fundamental question that emerges once we accept the validity of these explanations: does the Mexican-origin population living in the U.S. experience distributions of birthweight that are similar to those of their migrant peers in the U.S.? If the behavioral and network explanations hold, Mexican-origin groups living in the U.S. ought to have similar outcomes to Mexicans living in Mexico. Since we do not have access to national data for Mexico (or, for that matter, for any place in Central America that is a large sender of migrants to the U.S.), the comparison is difficult to make. Yet, it is highly unlikely that the low incidence of low birthweight among Mexicans in the U.S. is at all valid for Mexico as a whole for, if it were, it would imply exceedingly low levels of neonatal mortality, several times lower than those we observe at the national level. If this counterfactual is not true, how can one argue that factors endogenous to the Mexican social and cultural contexts provide an advantage to migrants, but are of negligible import in the absence of migration? In sum, the variable-based explanations come up short again because they ignore fundamental differences in context between individuals they are assuming to be the same-in this case, Mexicans living in the U.S and those living in Mexico.

\section{THE MATTER OF SELECTION}

Although the problems associated with the variable approach that undermine the existence of a paradox in both mortality and birthweight are all quite pertinent, we focus on only one of them, social selection. Selection effects can occur in a population-based health study whenever there is a criterion for entry into the sample that excludes some potential sample members on health-related grounds. Selection has received not more than passing attention as an alternative explanation to either paradox. This is in contrast with the attitude that researchers often have regarding selection problems in adult mortality and as a factor affecting the relations between SES and health status in general. It is paradoxical, for example, that although many investigators recognize that the black-white mortality cross-over has more than a passing chance of being due to heterogeneity in frailty, very few seriously consider that social selection may be the root explanation of the Hispanic in any of its incarnations.

In this section we use microsimulations to show how even a minor amount of selection can induce large biases in the estimation of ethnic differences in infant and adult health outcomes and, thus, mislead the investigator eager to trumpet the existence of a paradox. We first define several stages of the selection process, relate them to the existence of social effects, and show that the causal complexity associated with the paradox is more than what can be captured through conventional models. To simplify presentation, in what follows we assume that the Hispanic paradox pertains to comparisons between non-Hispanic whites and the Mexican-origin population.

\section{Social Selection Processes}

There are two selection mechanisms that could produce the appearance of better pregnancy outcomes among Mexican-origin mothers. The first involves the possible selection of Mexican migrants for superior health status. This includes processes 
whereby those who migrate to the U.S. are selected in places of origins and, equally important, processes that influence the rate of retention in the U.S. among those who succeed in securing temporary residence in the U.S. Successful migration and retention of residence in the U.S. require a number of preconditions about which we know little. Furthermore, whatever we know is never explicitly entered into models of health, mortality, or pregnancy outcomes. First, in all probability, those who attempt to migrate and stay are among the healthiest in the sending population. ${ }^{19}$ The journey is demanding, and successful residence requires considerable skills, levels of physical and psychological stamina, and even peculiar abilities to take on risks and simultaneously fend off associated deleterious effects of stress and uncertainty. These physical and mental health attributes could, in turn, also lead to more positive pregnancy outcomes. Second, it has been argued convincingly that successful migrants often belong to social networks that provide information, facilitate the move, and ease the processes of adjustment and adaptation. (These arguments about selection processes would be unchanged if instead of pregnancy outcomes we were referring to adult health and mortality.) These network factors could also be related to positive pregnancy outcomes.

The second mechanism of selection-associated with birth outcomes but not with child or adult mortality and health-operates through fetal deaths. Women must carry their pregnancies to term in order to be included in most sample designs. Women who experience any form of fetal loss, such as a miscarriage or an induced abortion, have been excluded from the analysis of birthweight in almost all previous studies. Different pathways to fetal loss (e.g., abortion and miscarriage) could bias the resulting sample in different ways with respect to the probability of a low birthweight outcome. For example, hypothetically, if women who experienced miscarriages had carried their pregnancies to term, they might have been likely to experience low birthweight. However, the same might not be true for women who had induced abortions- that is to say, they might not have been as likely to have had a low birthweight child if they had carried their respective pregnancies to term. Moreover, if the likelihood of experiencing pregnancy loss through one of these specific pathways is shaped, in part, by the ethnicity of the mother, then the issue of selection bias could have far-reaching implications for the birthweight paradox. For example, if women of Mexican-origin who are exposed to higher pregnancy risks also experience higher rates of fetal deaths than do women in the standard population, then the observed pregnancy and birth outcomes will be more favorable among the former than the latter.

This conjecture is plausible but difficult to test because it requires accurate records on all cases of fetal loss as well as data on the risk profiles for all women who become pregnant, not just of those who give birth. Preliminary findings from the Hispanic Health and Nutrition Examination Survey (HHANES) and NSFG undermine the hypothesis that selection due to fetal loss could explain the paradox because they reveal that rates of fetal deaths are lower among the three largest Hispanic groups, and are lowest for Mexican Americans. ${ }^{41,45}$ Other research, however, suggests that it is perhaps premature to rule out the contribution of this mechanism because rates of fetal deaths by parity display larger ethnic variability than previously thought, and appear to be unfavorable for Mexican Americans for pregnancies of third or higher order. ${ }^{22}$ Furthermore, information available on involuntary and voluntary abortions is notoriously deficient and cannot be totally relied upon to test our 
conjecture. Finally, if selection through fetal deaths operates simultaneously with the health selection of migrants, the potential size of effects is significantly reduced and effects of the former process is enhanced unless the healthiest of women are those who would be more likely to experience fetal deaths among migrants. For these reasons, we focus our analysis below on selection due to migration.

The literature on the Hispanic concerning birthweight (and infant and child health and mortality) is remarkably silent about the possible operation of selection processes (for an exception, see Landale et al. ${ }^{19}$ ). Furthermore, the usual claim made when admitting the possibility of selection, namely, that the researcher explicitly controls for factors that affect selection, can be profoundly misleading. This is because the usual control factors are attributes themselves affected by selection processes. As we show below, this only aggravates the bias induced by the original selection process. Thus, for example, there is a widespread belief that controlling for education manages to attenuate migration selection bias associated with estimated effects. However, since educational attainment in the place of origin is likely to be affected by health of the migrant, it is very possible that controls for education will induce even worse biases.

The state of affairs in the literature on the Hispanic paradox relating to adult health and mortality is not much different. We only know of a handful of examples where the dangers of selection for inferences regarding differentials in health and mortality are taken seriously and where there is an assessment of the role it may play. ${ }^{46,47}$

To what extent are these selection mechanisms deserving of attention? Is the magnitude and direction of the bias involved sufficiently large and insidious that we should care about them? It is widely known that selection produces biased and inconsistent estimates of effects in the context of general linear models, and there is a considerable amount of literature on procedures that purportedly attenuate or eliminate the problem (see, for example, Maddala ${ }^{48}$ ). Less is known, however, about the exact magnitude or direction of errors in general and, least of all, in the particular case that occupies us.

In this section we use Monte Carlo simulation to provide an idea of the importance of errors produced by the two selection mechanisms identified above. We start by posing the problem more formally, and then proceed to perform simulations to assess errors under scenarios representing varying degrees of selection. Although throughout we focus on pregnancy outcomes, the procedures and conclusions extend to the case when adult health and mortality are the outcomes of interest.

The most important results are that (1) even a small amount of health selection can produce the impression of better pregnancy outcomes among successful migrants and (2) conventional controls for selection can augment and enhance the bias.

\section{A Simple Example}

In this example, the process of migration, represented by outcome $O(1)$, is a binary variable and has two possible results: it attains the value 1 when successful (the individual migrates), and a value 0 when it is not successful (the individual does not migrate). The pregnancy outcome (in this case low birthweight), denoted by outcome $O(2)$, is also a binary variable, and it attains the value 1 for low birthweight 
and 0 otherwise. We observe $O(2)$ only among successful Mexican migrants to the U.S. (i.e., Mexican-origin mothers with $O(1)=1$ ) and among all non-Hispanic whites in the U.S. (which in this case is our comparison group).

The relations between the outcomes can be simply expressed in terms of four equations. To avoid cluttering we assume that there is only one underlying factor affecting migration, and that is health status. We have two sets of equations, one determining propensity to migrate and the other expressing the influence of health status and other factors on birthweight. The migration equations are as follows:

$$
\begin{gathered}
e_{1}=v_{1} \\
O(1)= \begin{cases}1 & \text { if } e_{1} \geq t_{1} \\
0 & \text { otherwise },\end{cases}
\end{gathered}
$$

where $e_{1}$ is a measure of health status, $v_{1}$ is a random $(0,1)$ normal variate representing unobserved health status, and $t_{1}$ is a threshold value that determines the amount of health selection among migrants (the greater the magnitude of $t_{1}$ the more important health status is for migration). Hence, $e_{1}$ represents the unobserved propensity to migrate, and it is assumed to be a function only of health status. We also have the following birthweight equations:

$$
\begin{gathered}
e_{2}=\lambda v_{1}+v_{2} \\
O(2)= \begin{cases}1 & \text { if } e_{2} \geq t_{2} \\
0 & \text { otherwise },\end{cases}
\end{gathered}
$$

where $e_{2}$ represents birthweight, $v_{1}$ is the random $(0,1)$ normal variate representing unobserved health status, $v_{2}$ is another random $(0,1)$ normal variate representing factors other than health that affect birthweight, $\lambda$ is the effect of unobserved health on the probability of a low birthweight baby, and $t_{2}$ is a threshold value that determines the amount of low birthweight in the population (the greater the magnitude of $t_{2}$, the higher the incidence of low birthweight). The correlation between $v_{1}$ (health status) and $v_{2}$ (non-health factors affecting birthweight) can be expressed as $\lambda /\left(\lambda^{2}+1\right)^{1 / 2}$. These relations imply that we observe pregnancy outcomes in the migrant group only if $v_{1} \geq t_{1}$. In other words, Equations (1) and (2) imply that if the unobserved characteristics representing health status $\left(v_{1}\right)$ are above a certain threshold level $\left(t_{1}\right)$, successful migration occurs. An analogous interpretation applies to Equations (3) and (4), only here the outcome is low birthweight. The corresponding error term, $e_{2}$, represents conditions affecting birthweight; it is partially correlated with maternal health status but also affected by unrelated factors, represented by $v_{2}$.

The key problem in this simple, one-stage selection process is that the observed realization of the second outcome (low birthweight) is conditioned by the behavior of the error term in the other equation (migration). If $O(2)$ were a linear variable (rather than a dichotomous one), we would express this by saying that the expected value of $O(2)$ in one group is a function of linear predictors and of the expected value of $e_{2}$, conditional on the fact that $e_{1} \geq t_{1}$ (i.e., that migration occurs), rather than on the linear combination of predictors in the birthweight equation alone. Even though $O(2)$ is a dichotomous rather than continuous variable, the idea holds the same: the behavior of $O(2)$ depends on two error terms $\left(v_{1}\right.$ and $\left.v_{2}\right) .{ }^{e}$ We can now formulate the 
question of interest to us: suppose that Mexican-origin women who are successful migrants to the U.S. are also more likely to be in better health than those who are not. What will be the influence of the correlated processes (values of $\lambda$ and $t_{1}$ ) on inferences regarding the relative incidence of low birthweight in the target and standard population?

We assume that the purpose of the analysis is to compare the fraction of low birthweight infants in two groups, a U.S. native (standard) group and a Mexican migrant (target) group. We start out with a hypothetical population of 6,000 individuals, of which exactly half are U.S. natives and the other half are potential migrants living in Mexico. Individuals are randomly assigned values for their underlying health status $\left(v_{1}\right)$. These values are drawn from normal distributions with means that are identical to each other or, alternatively, from distributions such that the mean for the Mexican population is always lower than for the U.S. population. The results of the simulations discussed here assume, first, that the means of the health distributions are identical in the native and migrant populations and, second, that the mean is -0.25 in the origin population where migrants are drawn from (rather than 0 as it is in the native population). We assign several values to $t_{1}$ and $\lambda$ and generate probit estimates of the effects of membership in the migrant group on the probability of low birthweight. We assume throughout that the processes whereby health influences birthweight are identical in both populations. Finally, the value of $t_{2}$ is set at -1.28 , securing a fraction of low birthweight equivalent to about 10 percent, slightly larger than what we observe in either the non-Hispanic or Hispanic population living in the US, and lower than observed in the black population. We repeat these simulations between 50 and 150 times.

FIGURE 3 displays the estimated effect of being a migrant relative to the standard population (Non-Hispanic whites) as a function of both $\lambda$ and $t_{1}$, for the case when the U.S. and Mexican migrant populations have identical means in the health distribution. The figure shows that the estimated effect for being in the Mexican migrant group is biased downward when either the threshold, $t_{1}$, or $\lambda$, the effect of this unobserved health status on low birthweight, increase. In other words, findings consistent with the paradox are most likely to appear both when there is more health selection in the migration process and when the aspects of health related to migration are also strongly associated with low birthweight. We also simulated the case when the mean of the health distribution among in the population of origin is -0.25 instead of 0 . This represents a scenario when the entire population of origin has worse health. Although the biases diminish, as they should, their magnitude is still considerable. Except for implausible cases, that is, when the distribution of health status in the origin population is more than two standard deviations removed from the health status of the population of destination, the magnitude of the biases is always significant.

${ }^{e}$ If $e_{1}$ and $e_{2}$ are unrelated quantities $(\lambda=0)$ or if $t \rightarrow-\infty$, estimation of the equation for $O(2)$ would require nothing more than consideration of that equation in isolation from the other-there is no need to know anything about $O(1)$ to understand $O(2)$. In other words, when birthweight depends on processes and conditions unrelated to those that influence migration outcomes, there is no extra payoff if we understand better how migration takes place. As suggested previously, we suspect that this scenario is unlikely to be the case because error terms may be related to each other as a result of unmeasured conditions that influence both processes. The magnitude of this dependency is measured by $\lambda$. 


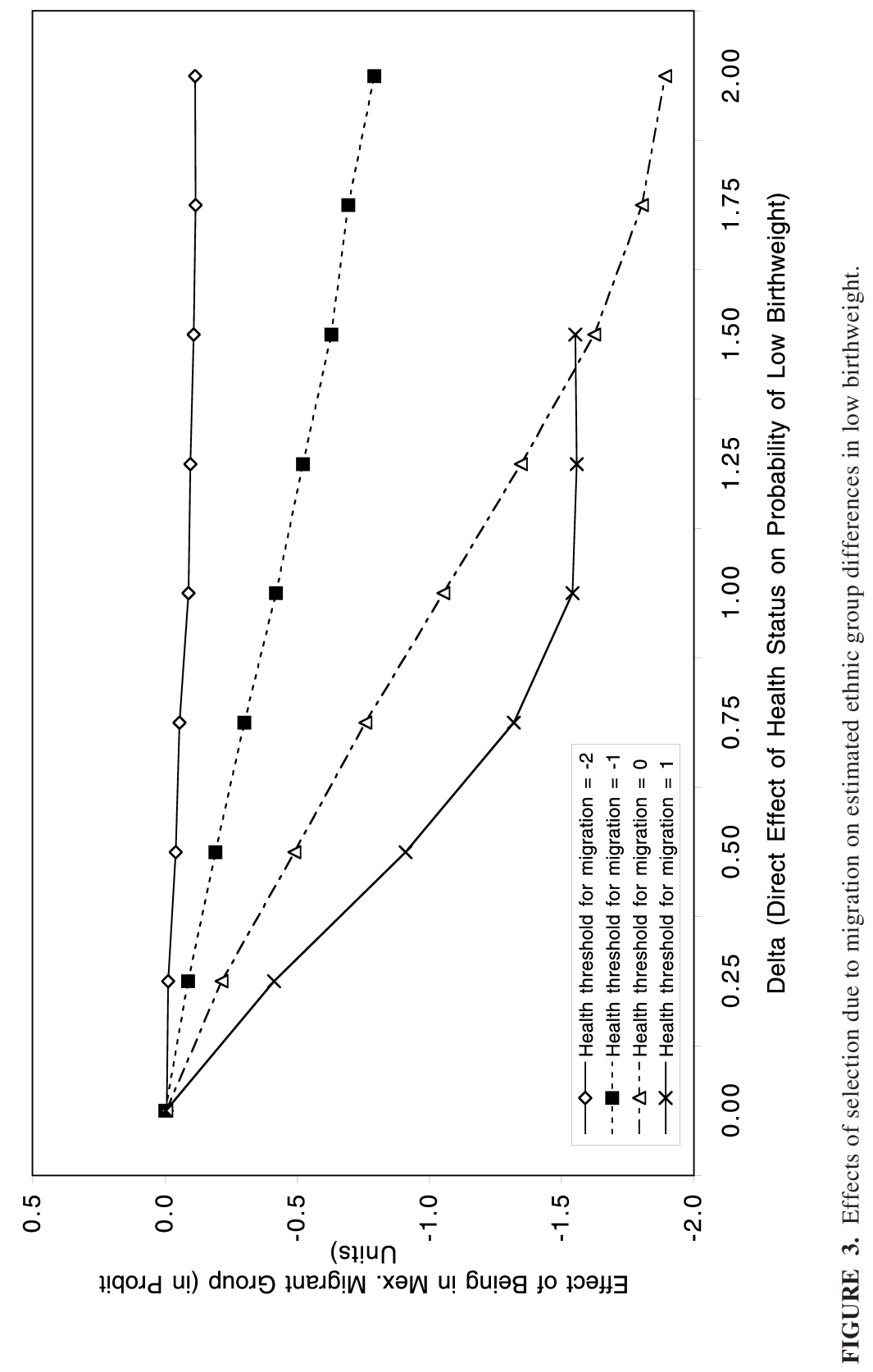


By construction the estimated effects should have a mean of 0 . However, because of the built-in selection the estimated effects always fall below 0. More negative values mean that members of the target group (migrants) have lower probabilities of low birthweight relative to the standard group. The size of the biases increase as $\lambda$ and $t_{1}$ increase.

Three important messages emerge from these simulations: (1) the correlation between maternal health and conditions affecting birthweight need not be too large for the bias to be sizeable, (2) the magnitude of health selection on migration need not be extreme for biases to be considerable, and (3) the larger the disparity in mean health conditions between the migrant and non-migrant group, the less relevant the selection process becomes.

\section{Making Matters Worse: Controlling for Selection}

Other than outright dismissal, the most frequent response to the charge that the Paradox is just a result of health selection to introduce "controls for selection". Among proponents of the linear model this solution can be justified as follows: to the extent that it is possible to compare birthweight distribution of the Mexican-origin population across categories of attributes that may account for successful migration, it should be possible to minimize the problems caused by selection biases. This line of thought is misleading: if the attributes the investigator is controlling happen to be a function of the same attributes that jointly determine migration and birthweight, controlling for them will not solve the problem and, worse yet, may even aggravate it.

Consider, for example a control for education. Linear models where education and ethnic group status variables appear side to side are commonplace. The justification for entering the control for education is both to account for the risk profile usually associated with lower education and to attenuate selection problems. However, suppose that people who attain higher levels of education while living in Mexico do so in part because they have superior physical and mental health, and that such positive health status also increases the risk of successful migration to the U.S. In this case, individuals with the highest levels of education are not just more likely to migrate, they are also more likely to have better pregnancy outcomes. Moreover, the relationship between health and education may be different in Mexico than it is in the U.S. if, for example, Mexican women must surmount more obstacles to obtain higher levels of education. In other words, higher educated women in Mexico could be more selectively healthy and motivated compared to women with less education than is the case in the U.S. If this is the case, comparisons between Mexican-origin women who migrate and whites of similar educational level will lead to an even larger overestimation of the effect of belonging to the migrant group. In short, controlling for education as a means of addressing the healthy migrant selection effect can lead to even bigger problems.

To assess the numerical magnitude of the biases when an erroneous control is introduced, we simulated four subpopulations as defined by the cross-classification of migrant/non-migrant and high/low levels of education. The model described above is now complicated by the introduction of a second outcome, attaining a high level of education. This requires that we specify the correlation between health and 
educational attainment in addition to the correlation between health and migration. Furthermore, we must also specify a threshold value determining the severity of health selection in education. To complete the model, we add an effect of education to the equation for birthweight, although this refinement does not change the main
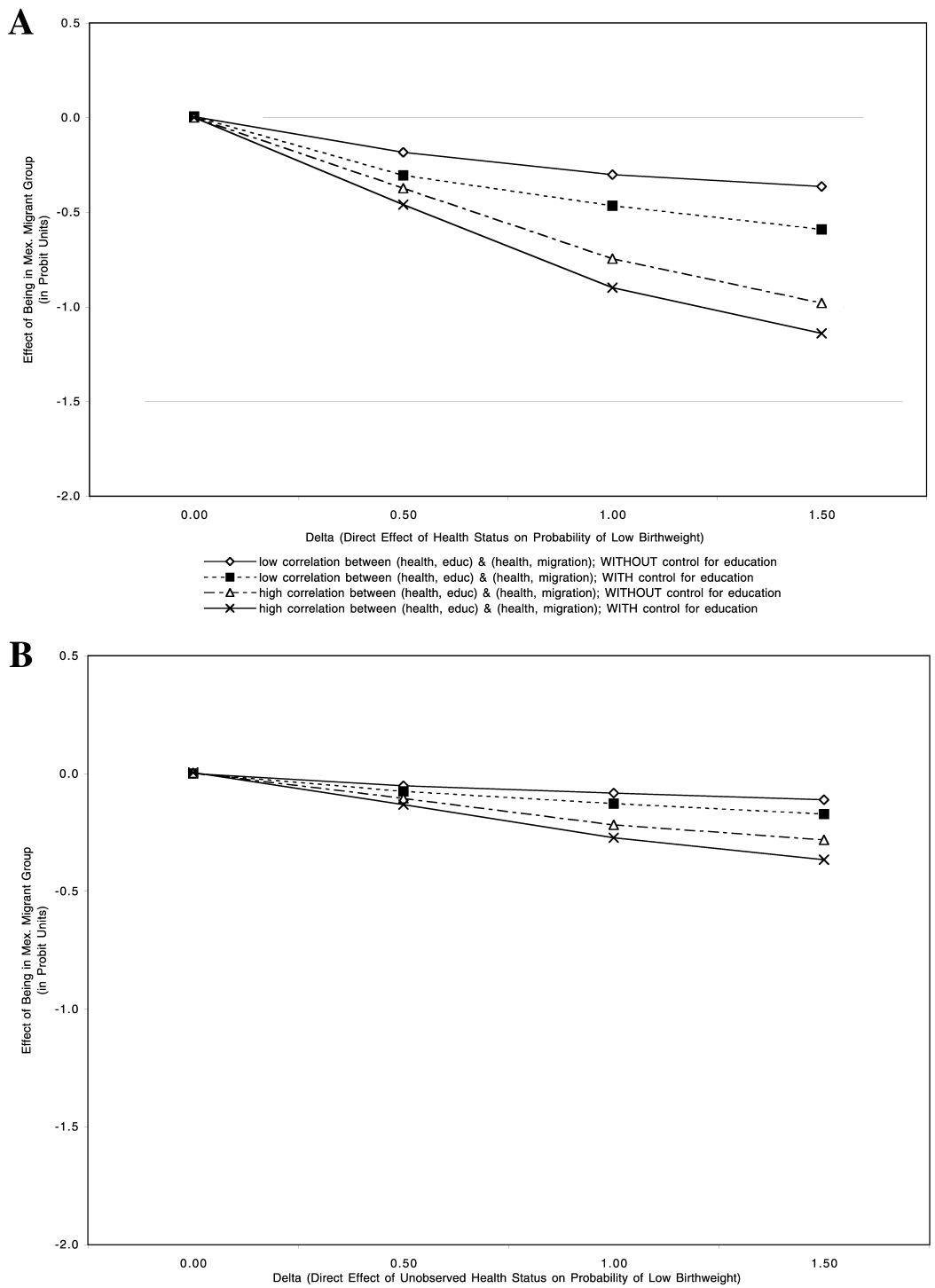

FIGURE 4. Effects of selection due to migration and education on estimated ethnic group differences in low birthweight with (A) high and (B) low health threshold for migration. 
results substantially. FIGURE 4 displays the estimated (probit) effects of belonging to the migrant group under different levels of severity of health selection into migration $\left(t_{1}\right)$. In Figure $4 \mathrm{~A}$ there is a high threshold for migration, meaning that only the healthiest 50 percent of Mexican women can migrate to the U.S. $\left(t_{1}=0\right)$. FiguRE 4B shows results corresponding to a low threshold for migration, one where migration is not as selective on health $\left(t_{1}=-1.25\right)$. The graphs display the probit effects estimated both with and without control for education and for selected combinations of values of the correlation between health and education and health and migration.

These figures show that the magnitude of errors in the estimated effect of ethnic group (measured as deviation from 0) increase (1) as the correlation between health and migration increases, (2) as the correlation between health and education increases, and (3) as the severity of health selection into migration education escalates. In addition, the biases are always larger when in the cases where there is a control for education than in cases where there is not The main lesson that one learns from these results is that unless a suitable model is used to interpret results, biases caused by selection can be aggravated when improper controls are chosen: far from being a safe heaven, the easy escape route routinely prescribed for the maladies caused by selection problems can turn into a fatal flaw. (To avoid cluttering the plots the figures discussed below do not include the estimated confidence interval associated with the mean values of the estimated effects in the simulations. Their inclusion and consideration does not alter any of the inferences we draw. They are available upon request.)

\section{ADULT HEALTH AND MORTALITY}

\section{Brief Review of the Problem}

The finding that migrant populations tend to show either similar or much better adult mortality experience than native populations is quite pervasive. Marmot et al. ${ }^{49}$ found that migrants in England have lower mortality than either persons born in England or those that remained in the sending country. Rogot and colleagues ${ }^{50}$ found that foreign-born persons who migrated to the U.S. have lower mortality than do U.S.-born individuals. In a previous study using birthplace statistics Kestenbaum ${ }^{51}$ detects a similar finding, namely, that those born outside the U.S. have lower mortality than U.S.-born individuals.

In a number of studies on mortality patterns among Puerto Ricans and other Hispanics living in the U.S., Rosenwaike ${ }^{51}$ and Rosenwaike and colleagues ${ }^{53}$ found systematic differences that favor the migrants over the U.S. born population. Although they did not perform a complete analysis-since they did not compare Hispanic population in the U.S. and corresponding sending Hispanic populations, the patterns they observed are fairly regular and consistent. This finding too is part of the so-called Hispanic Paradox.

Studies by Markides and colleagues ${ }^{54}$ and by Smith and Kington, ${ }^{55,56}$ review patterns of differentials in health status also and, although the evidence is more ambiguous there than in the case of mortality, they too detect a more favorable situation 
among Hispanic origin populations than among the US-native population. As was the case for early childhood health status and mortality, the Paradox can be a result of genuinely better health and mortality conditions among migrants, but it may also reflect the impacts of measurement errors and of selection. First, there is a real possibility that elderly Hispanics exaggerate their age. ${ }^{57}$ This alone imparts a downward bias on mortality rates and could explain in part at least the patterns of mortality advantages already identified. (Since a similar propensity has been found to affect black American adult mortality patterns ${ }^{42}$ there is less reason to suspect that age overreporting explains also why Hispanics adult mortality is advantageous relative to that of Blacks. Systematic age misreporting may also bias the observed results regarding health status. However, this is less likely since the information on which those patterns have been uncovered is characteristic for its good age reporting.) The mechanisms through which selection may operate among adults, are more complex that those affecting infant and child health and mortality and deserve close scrutiny. First, selection through migration may adopt the same form described before: individuals who reach the U.S. and become residents are likely to be drawn from a population that is less frail than the one that does not migrate. Depending on how rigorous selection is, this can go a long way toward explaining lower mortality and morbidity among U.S. immigrants. (Researchers in this area are well aware that selection is a serious competing explanation, though they do not explicitly test for it. ${ }^{58-60}$ )

However, there are also a number of complicating factors that need to be discussed. First, age at migration makes a difference: whereas selection on favorable health may be characteristic of those who are relatively young at the time of migration because of their shorter time of exposure to deleterious conditions in the sending area, those who migrate at relatively old ages may represent a stock of individuals whose longer exposure to deleterious conditions makes them relatively more frail. The distribution of migrants by age at migration is thus a crucial piece of information. Second, if the effect of early exposure to deleterious conditions does not manifest itself until later in life, then selection of healthier members at young ages will manifest itself in two regularities: better health and mortality profile soon after migration and a deteriorating profile as individuals age in the country of destination. These effects will mimic those derived from adaptation and assimilation that lead to embracing potentially harmful life styles.

In sum, unlike the case of infant and child health, the comparison of adult health and mortality between migrants and non-migrants is fraught with potential artifacts, some of which display patterns typical of health selection and others which move in opposite direction, offsetting initially beneficial effects associated with health selection. In the following section we assess the magnitude and direction of simple selection effects. The results we obtain probably overstate their magnitude since we ignore the operation of other, equally plausible mechanisms (such as those associated with late effects of early exposure). 


\section{Magnitude of Biases in Adult Mortality Estimates Associated with Health Selection}

As we did in the case of birthweight, we are able to calculate the magnitude and direction of biases that may affect estimates of adult mortality and health status when there is health selection of migrants. An exercise such as that we undertake here, has already been performed by Swallen ${ }^{46}$ for a number of migrant populations. Our effort differs only in that we provide a closed expression for the magnitude of the biases. Her conclusions are very similar to ours.

To simplify exposition, we concentrate on the case of mortality and narrow our inquiry to the case where the force of mortality for individual $i$ above some arbitrary age, say $x$, can be represented as follows:

$$
\mu_{i}(y)=\lambda_{i} \mu_{0}(y), \text { for } y>x \text { and } \lambda_{i}>0
$$

where $\mu_{i}(y)$ is a baseline hazard and $\lambda_{i}$ is an individual frailty factor for individual $i$, which, for simplicity, we assume to be gamma distributed with mean $\alpha / \beta$. It is well-known ${ }^{61}$ that under these conditions the average probability of surviving to age $z$ is given by:

$$
S(z)=\beta^{\alpha}(H o(z)+\beta)^{-\alpha}
$$

where $H o(z)$ is the integrated force of mortality up to age $z$. The average force of mortality at age $z$ is simply

$$
\mu(z)=\frac{-S^{\prime}(z)}{S(z)}
$$

where $S^{\prime}(z)$ is the first derivative of the survival function at age $z$.

Consider now the case of two subpopulations, one where values of $\lambda_{i}$ are drawn using the entire distribution, and one where individuals are drawn from a truncated distribution, say with $\lambda_{i}<\lambda_{0}$. The lower the value of $\lambda_{0}$ the more significant is migrant selectivity in terms of frailty. Under such conditions, one can show that the average probability of surviving to age $z$ among migrants, $S_{m}(z)$, is given by:

$$
S_{m}(z)=\frac{S_{n}(z) G_{1}\left(z, \lambda_{0}\right)}{G_{2}\left(z, \lambda_{0}\right)},
$$

where $S_{n}(z)$ is the average probability of surviving to age $z$ among non-migrants, $G_{1}\left(z, \lambda_{0}\right)$ is the distribution function of a gamma random variable with parameters $(\alpha, H o(z)+\beta)$ and $G_{2}\left(z, \lambda_{0}\right)$ is the distribution function of a gamma random variable with parameters $(\alpha, \beta)$.

One can show that, under these conditions, it will always be the case that $S_{m}(z)>S_{n}(z)$ for all $z$, except those at the tail end of the age span when survivorship drifts to 0 , and that therefore the mortality rate in the migrant population will be lower than in the non-migrant population. Unlike the case of birthweight, the bias associated with health selection of migrants will tend to vanish over time, as the migrants become older. This is because the composition by frailty of the migrant and nonmigrant population will converge toward each other and the initial truncation of frailty will become irrelevant. Thus, convergence of migrant and non migrants could be expected even in the absence of changes in behavioral profiles or exposure.

To illustrate the magnitude of the biases, FIgURE 5 A displays the ratios of mortality rates by five year age groups in the interval 30-80 that would be observed under different regimes of frailty truncation or health selection. FIGURE 5 B displays 

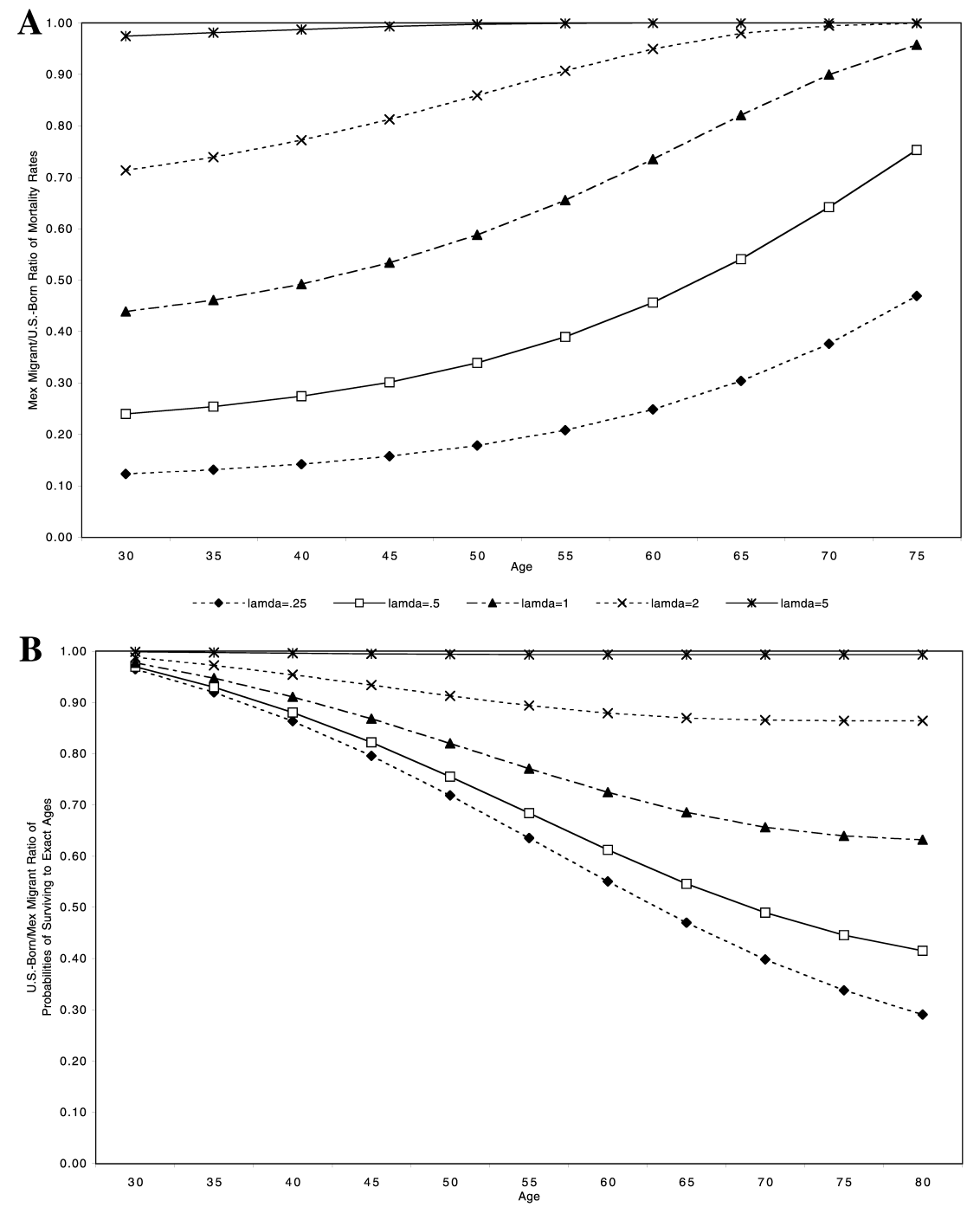

FIGURE 5. Effect of selection due to migration on estimated ethnic group differences in mortality: (A) mortality rates and (B) survival functions.

the corresponding survival curves. The underlying frailty distribution has a mean and variance equal to 1 . The most extreme regime in the graph is one where $\lambda_{0}=0.25$ and the most benign is one where $\lambda_{0}=5$. Note that in the first case the mortality rates among migrants we would observe are less than half the magnitude than among nonmigrants, and that even in a regime where selection is relatively minor (when $\lambda_{0}$ is very small) the biases can be quite substantial particularly at younger ages. 
It is important to note that these results suggest a way to identify health selection effects: indeed, if the age pattern of migrant mortality tends to converge toward the pattern of non-migrant mortality, there is prima facie evidence of health selection associated with migration. If, on the other hand, the cross over occurs but and at younger ages, it is more likely that reporting errors may the root cause (or, alternatively, persistence of cohort effects).

\section{WHERE TO GO FROM HERE?}

The purpose of this paper was to illustrate a few of the consequences associated with the application of the variable or risk approach. We used the example of the Hispanic Paradox pertaining to infant mortality and birthweight and made a brief incursion within the area of adult mortality. We showed the consequences of neglecting or considering only lightly social selection processes inherent in the definition of ethnic group the effects of which are sought out in all the analyses that uncover a paradox. We showed that the biases can be large even under benign conditions, that conventional remedies could result in further damage to the estimates, that nested selection processes do not ordinarily lead to gains in precision and, finally, that the entire enterprise of controlling for confounding influences, so fundamental in a risk or variable-based approach, can be self-defeating.

This high sensitivity of estimates to unobserved social selection processes has one important implication: given the already fragile results found in the literature, one cannot take the claims made in support of the Hispanic Paradox as an unassailable phenomenon. Indeed, the entire foundation on which the Paradox is built appears to crumble if only mild social selection is in place. In the case of early mortality, the selection perspective makes even more sense when one considers that estimates of neonatal mortality of the migrant population and the population of origin are so dramatically different and that migration theories insist on the importance of selection factors operating in migration (at least those that work via integration into social networks, which can produce similar biases).

These conclusions carry over verbatim to the Hispanic Paradox as it pertains to adult health and mortality as well. Not only is the fragility of extant estimates of the contrasts between Hispanics and non-Hispanic whites as extreme as it is in the case of birthweight or infant mortality but, in addition, the explanation invoking reporting errors (of mortality or health status) cannot be as summarily dismissed as it is in the case of infant mortality or birthweight. Thus, mild distortions due to selection combined with mild reporting errors may produce the appearance of a paradox when there is none at all. The case for the Paradox would fizzle rapidly here as well, for the impacts of selection are exactly analogous to those inherent in early health status and mortality.

So, where do we go from here? Further studies following the conventional risk or variable approach will produce only vapid stories, suffering from the same fragility as already expounded. What we need are more daring study designs and a less rigid adherence to the conventional linear models. First, we need study designs that integrate conditions affecting migration to the U.S. and those that regulate and constrain the lives of migrants who reside in the U.S. Inevitably this requires a historical approach, meaning either a longitudinal or, less satisfactorily, a retrospective study. 
Second, studies of the migrant population in the U.S. alone are not sufficient to draw believable inferences, so we should expand the lens of the study to involve the sending populations, including those people who have returned after being migrants, those who could have been migrants but were not, and those who tried unsuccessfully to be migrants. Third, because the processes of social selection and migration and those involving adaptation to the U.S. are likely to vary across groups and geographic locations, we cannot afford to overlook the extent to which the outcomes and the relation to attributes are a function of the social and historical space occupied by migrants. The study, per force, must consider high-order interaction effects between behaviors and social contexts. Hierarchical linear models will help but will not be enough. They introduce rigidities of their own. We need better models to capture interactor influences and to understand facilitators and obstacles to diffusion processes. These are important in the study of health also since they are an integral part of how individuals behave and influence each other, how do they access social relations for support, and how do they choose the environments where they live. It would indeed be paradoxical if these processes had no relation to health status. Until studies begin to adhere to these guidelines, the Hispanic Paradox, in any of its guises, should be a motivator for further research, not a punch line.

\section{REFERENCES}

1. Krieger, N. 1994. Epidemiology and the web of causation: has anyone seen the spider? Soc. Sci. Med. 39(7): 887-903.

2. Susser, M. \& E. Susser. 1996. Choosing a future for epidemiology. Am. J. Public Health 86(5): 668-677.

3. Sнy, C.M. 1997. The failure of academic epidemiology: witness for the prosecution. Am. J. Epidemiol. 145(8): 479-484.

4. Susser, M. 1998. Does risk factor epidemiology put epidemiology at risk? Peering into the future. Epidem. Comm. Health 52: 608-611.

5. Schwartz, S. \& E. Susser. 1999. A future for epidemiology? Annu. Rev. Public Health 20: 15-33.

6. Palloni, A., S. Kanaiaupuni \& M. Ceballos. 2001. The Hispanic paradox of low birth weight: an exegesis and metaanalysis. Forthcoming.

7. Casterline, J. \& B. Cohen. 2001. Diffusion Processes and Fertility Transition, Selected Perspectives. National Research Council, Washington, D.C.

8. Аввотт, A. 1988. Transcending general linear reality. Sociol. Theory 6: 169-186.

9. Аввотт, A. 1997. Of time and space: the contemporary relevance of the Chicago school. Social Forces 75(4): 1149-1182.

10. Ragin, C. 1987. The Comparative Method: Moving Beyond Qualitative and Quantitative Strategies. University of California Press, Berkeley.

11. Lieberson, S. 1985. Making It Count. University of California Press, Berkeley.

12. Ryder, N. 1964. The process of demographic translation. Demography 1(2): 74-82.

13. NI, B.M. 1992. A critique of the cohort approach to fertility. Pop. Devel. Rev. 18(4): 599-630.

14. Аввотт, A. \& A. Tsay. 2000. Sequence analysis and optimal matching methods in sociology: review and prospect. Sociol. Meth. Res. 29(1): 3-33.

15. Аввоtт, A. \& E. Barman. 1997. Sequence comparison via alignment and Gibbs sampling. Sociol. Meth. 27: 47-87.

16. Blume, L. \& S.N. Durlauf. 2001. The interactions-based approach to socioeconomic behavior. In Social Dynamics, S. \& H.P. Young Durlauf, Eds. MIT and Brookings Institutions..

17. Manski, C.F. 2000. Economic analysis of social interactions. National Bureau of Economic Research, March. Working Paper 7580. 
18. Palloni, A. 2001. Diffusion in sociological analysis: how useful is it for the study of fertility and mortality? In Diffusion Processes and Fertility Transition, Selected Perspectives. J. Casterline \& B. Cohen, Eds. National Research Council, Washington, D.C.

19. Landale, N., R.S. Oropesa \& B.K. Gorman. 2000. Migration and infant death: assimilation or selective migration among Puerto Ricans? Am. Sociol. Rev.

20. Teller, C. \& S. Clyburn. 1974. Trends in infant mortality. Texas Bus. Rev. 48(10): 240-246.

21. Gutmann, M.P., W. Parker Frisibie, P. DeTurk \& K.S. Blanchard. 1998. Dating the origins of the epidemiologic paradox among Mexican Americans. The University of Texas at Austin.

22. Frisbie, W.P. 1993. Infant mortality among Mexican Americans: 1935-1985. Final Report. National Institute of Child Health and Human Development.

23. Frisbie, P. 1996. The enigma of Mexican-origin pregnancy outcomes. Hispanic Maternal Child Health. NICHD and MCHB, Washington D.C.

24. BECERRA, J.E., et al. 1991. Infant mortality among Hispanics: a portrait of heterogeneity. J. Am. Med. Assoc. 265: 217-221.

25. CRAMER, J.C. 1987. Social factors and infant mortality: identifying high-risk groups and proximate cases. Demography 24: 299-322.

26. Moss, N. \& K. CARver. 1992. Explaining racial and ethnic differences in birth outcomes: the effect of household structure and resources. Pop. Assoc. Am. Denver.

27. Rogers, R.G. 1989. Ethnic differences in infant mortality: fact or artifact? Soc. Sci. Quart. 70: 642-649.

28. Williams, R.L., N.J. Binkin \& E.J. Clingman. 1986. Pregnancy outcomes among Spanish surname women in California. Am. J. Public Health 76: 387-391.

29. Forbes, D. \& W.P. FrisbiE. 1991. Spanish surname and anglo infant mortality: differentials over a half-century. Demography 28: 639-660.

30. Hummer, R.A., I.W. Eberstein \& C.B. NAM. 1992. Infant mortality differentials among Hispanic groups in Florida. Social Forces 70: 1055-1075.

31. Rumbaut, R.G. \& J.R. Weeks. 1991. Perinatal risks and outcomes among low-income immirgrants. Final report for the Maternal and Child Health Research Program. Rockville: Department of Health and Human Services.

32. Ventura, S.J. 1985. Births of Hispanic parentage, 1982. Monthly Vital Stat. Report 34: 4.

33. Collins, J.W. \& D.K. Shay. 1994. Prevalence of low birth weight among Hispanic infants with U.S.-born and foreign-born mothers: the effect of urban poverty. Am. J. Epidemiol. 139: 184-192.

34. SCRIBNER, R.A \& J.H. Dwyer. 1989. Acculturation and low birth weight among Latinos in the Hispanic HANES. Am. J. Public Health 79: 1263-1267.

35. SCRIBNER, R.A. 1991. Infant mortality among Hispanics: the epidemiological paradox. J. Am. Med. Assoc. 265: 2066.

36. CoBAs, J.A., et al. 1996. Acculturation and low birthweight infants among Latino women: a reanalysis of HHANES data with structural equation models. J. Public Health 86: 394-396.

37. James, S. 1993. Racial and ethnic differences in infant mortality and low birth weight: a psychosocial critique. Ann. Epidemiol. 3: 130-136.

38. SHINO, P.H., et al. 1986. Birth weight among women of different ethnic groups. J. Am. Med. Assoc. 255: 48-52.

39. Bean, F.D. \& M. Tienda. 1987. The Hispanic population of the United States. Russel Sage Foundation, New York.

40. Hauser, R.M., S. Solomon \& D. Pager. 2001. High school dropout, race-ethnicity, and social background from the 1970s to the 1990s. University of Wisconsin-Madison, Working Paper.

41. Morenoff, J.D. 2000. Unraveling Paradoxes of Public Health: Neighborhood Environments and Racial/Ethnic Differences in Birth Outcomes. Ph.D. Diss., University of Chicago, Chicago.

42. Preston, S.H., et al. 1996. African-American mortality at older ages: results of a matching study. Demography 33(2): 193-209. 
43. Sherraden, M. \& R.E. Barrera. 1994. Mexican immirgrant women and childbearing: poverty, family support, and birth weight. Midwest Sociological Society, St. Louis.

44. Guendelman, S. \& B. Abrams. 1995. Dietary intake among Mexican American women: generational differences and a comparison with white non-Hispanic women. Am. J. Public Health 85: 20-25.

45. Pletsch, P.K. 1988. Hispanics: at risk for adolescent pregnancy? Public Health Nursing 7: $105-110$.

46. Swallen, K.C. 1997. Do health selection effects last? A comparison of morbidity rates for elderly adult immigrants and US-born elderly persons. J. Cross-Cultural Geronotol. 12(4): 317-339.

47. Swallen, K.C. 1998. Mortality and nativity: a consideration of race, ethnicity and age at time of immigration. Unpublished Manuscript.

48. Maddala, G.S. 1983. Limited Dependent and Qualitative Variables in Econometrics. Cambridge University Press, New York.

49. Marmot, M.G., A.M. Adelstein \& L. Bulusu. 1984. Lessons from the study of immigrant mortality. Lancet 1: 1455-1457.

50. Rogot, E., P.D. Sorlie, N.J Johnson \& C. Schmitt. 1992. A study of 1.3 million persons by demographic, social and economic factors: 1979-1985 follow-up. National Institute of Health Publication No. 92-3297.

51. Rosenwaike, I. 1987. Mortality differentials among persons born in Cuba, Mexico and Puerto Rico residing in the United States, 1979-1981. Am. J. Public Health 77(5): 603-606.

52. Rosenwaike, I., Ed. 1991. Mortality of Hispanic Populations. Greenwood Press, New York.

53. Markides, K.S., L. Rudkin, R.J. Angel \& D.V. Espino. 1997. Health status of Hispanic elderly. In Racial and Ethnic Differences in the Health of Older Americans. L.G. Martin \& B.J. Soldo, Eds.: 285-300. National Academy Press, Washington D.C.

54. Smith, J.P. \& R.S. Kington. 1997. Demographic and economic correlates of health in old age. Demography 34(1): 159-170.

55. Smith, J.P. \& R.S. Kington. 1997. Race, socioeconomic status, and health in late life. In Racial and Ethnic Differences in the Health of Older Americans. L.G. Martin \& B.J. Soldo, Eds.: 105-162. National Academy Press, Washington D.C.

56. Dechter, A. \& S.H. Preston. 1991. Age misreporting and its effects on adult mortality estimates in Latin America. Pop. Bull. United Nations 31-32: 1-16.

57. Markides, K.S. \& J. Coreil. 1986. The health of Hispanics in the Southwestern United States: an epidemiologic paradox. Public Health Rep. 101(3): 253-265.

58. Abraido-Lanza, A.F., D.P Dohrenwend, D.S NG-MaK \& J.B. Turner. 1999 The Latino mortality paradox: a test of the "salmon bias" and healthy migrant hypotheses. Am. J. Public Health (October) 1543-1548.

59. Sorlie, P.D., E. Backlund, N.J. Johnson \& E. Rogot. 1993. Mortality by Hispanic status in the United States. J. Am. Med. Assoc. 270(20): 2464-2468.

60. Vaupel, J., K.G. Manton \& E. Stallard. 1979. The impact of heterogeneity in individual frailty of the dynamics of mortality. Demography 16: 439-454. 